\title{
An unusual presentation of generalized tetanus as dysphagia
}

\author{
Powthira. $R^{1}$, Peranantharajah. $T^{1}$, Nisahan. $B^{1}$
}

Teaching hospital, Jaffna.

\section{Introduction}

Tetanus is a disease caused by the toxin produced by Clostridium tetani, a Gram negative anaerobic spore forming bacillus. Infection acquired from body piercing and puncture wound; intravenous drug abuse, surgical interventions, septic abortion and through infected umbilical stump. After inoculation C.tetani transform into a vegetative rod shaped bacterium and produces metalloproteases such as tetanospasmin and tetanolysin. Tetanospasmin is responsible for painful muscle rigidity and autonomic instability via inactivating inhibitory neurotransmission. The role of tetanolysin is not known.(1)

\section{Case presentation}

A 68 year old previously healthy farmer from poor socioeconomic background admitted with painful difficulty in swallowing of one day duration. Dysphagia was noted for both the solid and liquids. He had no history of fever, malaise or headache. There were no history of throat injury or significant weight loss in the recent past. Family history was unremarkable. His initial symptoms were quickly worsened to painful spasms of neck and back muscle. He had never undergone any surgical interventions.

His GCS was normal, well oriented and responded appropriately. He was afebrile with normal pulse rate and blood pressure. His throat examination was normal. He had no lymphadenopathy. His respiratory and central nervous system examinations were normal. There was a prick injury noted at his left big toe. The wound was clean without any signs of inflammation. Detailed evaluation of wound revealed that one week ago he had a history of thorn prick injury. There was no history of bleeding or pain at the injury site. He did not seek any medical attention for that prick injury and he never had tetanus immunization.

The basic investigations such as full blood count, renal functions and liver functions were normal.
Inflammatory markers such as C-reactive protein and Erythrocyte Sedimentation Rate were also normal.

Clinical diagnosis of tetanus was made and he was monitored in Intensive Care Unit(ICU). The ICU care was given in isolation room with minimal disturbances. Midazolam infusion was given to reduce the spasms. To reduce the bacterial load oral Metronidazole was administered for 10 days. Tetanus immunoglobulin (TIG) 3000 Units was given intramuscularly in multiple sites and around the wound. Following day his spasms got worse and suddenly went into respiratory arrest and ventilatory support was given. He developed fluctuating blood pressure and tachycardia. Inotropic support as well as GTN infusion were needed to support the hypotension and hypertension respectively. Moreover Magnesium Sulphate $2 \mathrm{~g}$ bolus was given over $30 \mathrm{~min}$ followed by $2 \mathrm{~g}$ per hour intravenous infusion. Early Tracheostomy was performed and ventilatory support continued for 40 days. His ICU stay was complicated further with ventilator associated pneumonia and his blood culture was positive for E-coli and tracheal aspiration was positive for Acinatobacter. Sensitive antibiotics and physiotherapy were given appropriately. After seven weeks he fully recovered from illness and Tetanus toxoid was given.

\section{Discussion}

There are four types of tetanus. One is local tetanus which presents with tonic and spastic muscle in one extremity or body part. Second one is cephalic tetanus which initially involves only cranial nerves and later may develop as generalized tetanus. Third one is neonatal tetanus which is usually by contaminated umbilical stump. Forth one is generalized tetanus which is the most common and severe form.

The diagnosis of generalized tetanus is solely based on clinical history and physical examination. Disease severity can be predicted by assessing 
the incubation period.(2) The widespread distribution of C. tetani spores in our environment in combination with the lack of herd immunity leads to incidental tetanus infections in nonimmunized individuals.(3) This case report urges on considering generalized tetanus infection, when confronted with non-immunized man who presented with unexplained oropharyngeal symptoms.

Oropharyngeal symptoms in generalized tetanus is very rare and is reported in two case reports. $(4,5)$ In both cases, the patients presented with isolated symptoms of dysphagia and trismus, and generalized tetanus infection was recognized later. Our patient initially admitted to surgical unit with odynophagia and dysphagia later he developed neck and back muscle rigidity and eventually ended up in respiratory arrest.

Appropriate treatment of generalized tetanus consists of halting toxin production, neutralization of free circulating tetanus toxin, airway management, control of muscle spasm, management of dysautonomia and general supportive management. All patients should undergo wound debridement. Pharmacological eradication of $C$. tetani bacilli can be achieved by either Penicillin or Metronidazole based regimens. Metronidazole is the preferred choice. Human tetanus-specific immunoglobulin is available for neutralization of tetanospasmin which is the main cause for symptoms. Usual dose is 3000 to $6000 \mathrm{U}$ around the wound and as intramuscular injection (6). A recent meta-analysis showed that a combination of intramuscular and intrathecal Tetanus immunoglobulin (TIG) administration is superior to intramuscular treatment alone with regard to morbidities from tetanus. The most important factor to reduce mortality from generalized tetanus is care in the intensive care units (ICU) with aggressive sedation protocols and advanced ventilatory support. Benzodiazepine derivatives are the mainstay for sedation in the ICU during the course of generalized tetanus. Autonomic dysfunction remains the major clinical challenge; as hypotension, arrhythmia and cardiac arrest are important predictors of fatality. Magnesium Sulphate is known to reduce the duration of mechanical ventilation and autonomic instability. Since tetanus is one of few bacterial disease it does not confer immunity following recovery from acute illness, all patients with tetanus should receive active immunization with tetanus toxoid.(7)

\section{Conclusion}

The diagnosis of generalized tetanus remains a diagnostic challenge when the classical symptoms are absent at presentation. Early recognition and immediate initiation of advanced critical care is necessary to prevent rapid clinical deterioration and worst outcome. Though tetanus vaccination is well established in Sri Lanka, people from poor socioeconomic background we still unaware about the importance of vaccination. We recommend when evaluating the dysphagia in paliers with poor socioeconomic background, tetanus should be considered as an important differential after excluding the common causes of dysphagia.

\section{References}

1. Groleau G. Tetanus. Emerg Med Clin North Am 1992;10:351-60.

2. Brook I (2008) Current concepts in management of Clostridium tetani infection. Expert Rev Anti Infec Ther $6: 327-336$

3. Parker J (2006) Managing Tetanus. Emergency nurse 14: 14-17

4. Tahery J,Morris DP, Birzgalis AR: Tetanus: the 'forgotten disease'. A rare cause of dysphagia and trismus. J Laryngol Otol. 2004, 118: 974-976

5. Paterson AW, Ryan W, Rao Mudiginda VV: Trismus: or is it tetanus? A report of a case. Oral slug Oral med Oral Pathol Oral Radiol Endod. 2006, 101: 437-441

6. (2004) Tetanus Immune Globulin (Human). Bayer Corporation, Elkhart, IN, USA

7. Bleck TP. Clostridium tetani. In: Mandell GL, Bennett JE, Dolin R,eds. Principles \& practices of infectious disease. New York: Churchill Livingstone, 1995: 2173-8 